\title{
Desmopressin Lyophilisate for the Treatment of Central Diabetes Insipidus: First Experience in Very Young Infants
}

\author{
Kathleen De Waele ${ }^{1}$; Martine Cools ${ }^{1}$; Ann De Guchtenaere ${ }^{2}$; Johan Van de Walle ${ }^{2}$; Ann Raes ${ }^{2}$; \\ Sara Van Aken ${ }^{1}$; Kris De Coen ${ }^{3}$; Piet Vanhaesebrouck ${ }^{3}$; Jean De Schepper ${ }^{1,4, *}$ \\ ${ }^{1}$ Department of Paediatric Endocrinology, University Hospital Ghent, Ghent, Belgium \\ ${ }_{3}^{2}$ Department of Pediatric Nephrology, University Hospital Ghent, Ghent, Belgium \\ ${ }^{3}$ Department of Neonatology, University Hospital Ghent, Ghent, Belgium \\ 4 Department of Paediatric Endocrinology, University Hospital Brussels, Brussels, Belgium \\ ${ }^{*}$ Corresponding author: Jean De Schepper, Department of Pediatrics, Division of Paediatric Endocrinology, University Hospital Ghent, Ghent, Belgium. Tel: +32-93322760, Fax: +32- \\ 93323875, E-mail: jean.deschepper@uzgent
}

Received: January 12, 2014; Revised: April 26, 2014; Accepted: May 23, 2014

\begin{abstract}
Introduction: In neonates and small infants, early diagnosis of central diabetes insipidus (CDI) and treatment with desmopressin in low doses (avoiding severe hypo- or hypernatremia) are important to prevent associated high morbidity and mortality in this particular age group.

Case Presentation: We described pharmacokinetic and pharmacodynamic results of the use of recently launched oral desmopressin lyophilisate (Minirin Melt®) in two infants with CDI, diagnosed at the age of 12 and 62 days, respectively. We observed that a starting dose of $60 \mu \mathrm{g}$ of Minirin Melt $\circledast$ in the first case resulted in a pharmacokinetic profile largely exceeding the reference frame observed in children with nocturnal enuresis, while a dose of $15 \mu \mathrm{g}$ in the second case resulted in acceptable concentrations. After initial dose adjustments, administration of sublingual lyophilisate resulted in rather stable serum sodium concentrations.

Conclusions: Using Minirin Melt $₫$ in infants with CDI appears to be effective, easy to use and well tolerated.
\end{abstract}

Keywords:Desmopressin; Diabetes Insipidus; Infant; Hypernatremia

\section{Introduction}

Central diabetes insipidus (CDI) is very rare in (premature) neonates and infants (1). Etiological factors are idiopathic, asphyxia, cerebral infections (usually meningitis, encephalitis or ventriculitis), congenital anomalies of all types (holoprosencephaly, septo-optic dysplasia, encephalocoele) and rarely intraventricular hemorrhage $(2,3)$. Early diagnosis and treatment are important to prevent severe morbidity and mortality. Treatment in neonates and infants differs slightly because their diet contains a proportionally larger quantity of water. Careful and precise administration of small doses of synthetic analogue of vasopressin, desmopressin (dDAVP) is warranted to prevent potential severe side effects as hyponatremia and convulsions secondary to water intoxication (4). However, there is no appropriate infant formulation available allowing titration and individualization of treatment at low doses. Clinicians have experimented with subcutaneous administration of desmopressin and dilution of commercially available nasal solution, but there is a high variability in absorption of these formulations and stability studies are lacking. Some authors suggest that aiming at an un-physiological steady state with a higher plasma osmolality level, might be preferable to unstable plasma osmolality shifts during intranasal administra- tion of desmopressin. Desmopressin oral lyophilisate (Minirin Melt $®$ ) has been launched in 2005, mainly for the treatment of enuresis nocturna. It is a wafer-like formulation, which dissolves instantly in the mouth with no need for water. Its bioavailability is approximately $60 \%$ greater than tablets and retains similar levels of efficacy and safety at lower dosing levels than the tablet. We reported our experience with oral lyophilisate form (Minirin Melt $®$ ) in two very young infants with central diabetes insipidus.

\section{Case Presentation}

Case one was a preterm male infant, born by spontaneous vaginal delivery after a pregnancy of 28 weeks and 3 days with a weight of 1220 grams and length of $38.6 \mathrm{~cm}$. At 12 days of age, Enterococcus meningitis/ventriculitis was diagnosed, which was managed with intravenous antibiotics and supportive management. A few days later, he developed hypernatremia $(160 \mathrm{mmol} / \mathrm{L})$ and polyuria $(13 \mathrm{~mL} / \mathrm{Kg} / \mathrm{h})$ with normal glycaemia. Adequate hydration was performed to normalize the sodium levels, but during an episode of catheter sepsis with Staphylococcus aureus, serum sodium concentrations remained high (164 $\mathrm{mmol} / \mathrm{L}$ ) with a serum osmolality of $320 \mathrm{mOsm} / \mathrm{Kg}$ and polyuria with a urine osmolality of $60 \mathrm{mOsm} / \mathrm{Kg}$. Central

Copyright ( 2014 , Research Institute For Endocrine Sciences and Iran Endocrine Society; Published by Kowsar Corp. This is an open-access article distributed under the terms of the Creative Commons Attribution License, which permits unrestricted use, distribution, and reproduction in any medium, provided the original work is properly cited. 
diabetes insipidus was confirmed by administration of $60 \mu \mathrm{g}$ Minirin Melt@, which lowered the serum osmolality from $298 \mathrm{mOsm} / \mathrm{kg}$ to $259 \mathrm{mOsm} / \mathrm{kg}$. Urine osmolality increased from 113 to $430 \mathrm{mOsm} / \mathrm{Kg}$ within three hours of administration and persisted above $400 \mathrm{mOsm} / \mathrm{Kg}$ for 24 hours. At different time points, blood sample was obtained to assess the circulating concentrations of desmopressin. The concentration-time curve of a dose of 60 $\mu \mathrm{g}$ at the age of 20 days and a body weight of 1300 grams showed very high dDAVP concentration levels, persisting for more than 24 hours, demonstrating that even in critical ill children, bioavailability of melt formulation is largely maintained (Figure 1). On treatment with Minirin Melt@, serum and urine electrolytes and osmolality were monitored closely, in addition to weight and urine output. High variability in urine output and body weight necessitated initially several dose adjustments (by 15 $\mu \mathrm{g})$. At discharge, after 125 days, Minirin Melt $\AA 60 \mu \mathrm{g}$ was given daily, together with a normal fluid intake. The parents were taught to monitor body weight daily and to allow a short time of diuresis. This regimen resulted in stable random sodium concentrations, around 140 to $150 \mathrm{mmol} / \mathrm{L}$. Psychomotor development was severely delayed, and seizures and bilateral blindness were present. At the age of 12 months, MRI showed absence of bright spot, confirming the permanency of diabetes insipidus. At the age of 2.5 years, diabetes insipidus was managed with Minirin Melt $®, 60 \mu \mathrm{g}$ daily and combined oral and gastric feeding.

The second neonate was a girl, born at term by spontaneous vaginal delivery with a birth weight of 2700 grams and length of $47 \mathrm{~cm}$. Her mother, a 16-year old teenager, denied any use of medication or (illegal) drugs during pregnancy. At birth, mild dysmorphic features were noticed, including asymmetry of the skull and ptosis of the right eye lid (due to N. oculomotorius paralysis). Brain MRI revealed bilateral subdural hygroma, schizencephaly with polymicrogyria and midline abnormalities (absent septum pellucidum, agenesis of the corpus callosum, hypoplastic optic nerves and chiasm, and absence of normal posterior pituitary bright spot). On day 60, she was admitted to hospital for neurosurgical intervention with subdural peritoneal shunting. Her body weight was $4020 \mathrm{~kg}$ and her length was $52 \mathrm{~cm}$. Laboratory investigations on day 1 after the operation showed hypernatremia (168 mmol/L) with a serum osmolality of $322 \mathrm{mOsm} /$ $\mathrm{Kg}$ and a urine osmolality of $100 \mathrm{mOsm} / \mathrm{Kg}$. Careful reviewing of the history revealed that the infant was very hungry and irritable since shortly after birth, with very frequent soaked diapers and drinking more than 230 $\mathrm{mL} / \mathrm{kg} /$ day of formula. Nevertheless, she gained weight poorly. Normalization of urine output and osmolality after IV administration of dDAVP confirmed the diagnosis of central diabetes insipidus. Treatment with Minirin Melt@ $15 \mu \mathrm{g}$ was started the following day. Urine osmolality increased within three hours and remained above $400 \mathrm{mOsm} / \mathrm{Kg}$ for the next 12 hours. Pharmacokinetic data are represented in Figure 1. At the age of 12 months, she was well managed with Minirin Melt $₫ 45 \mu \mathrm{g}$ daily. No episode of hypo or extreme hypernatremia occurred and a normal weight gain was observed. A severe neurological impairment was documented. At the age of 2.5 years, diabetes insipidus was managed with Minirin Melt@, 45 $\mu \mathrm{g}$ daily and gastric feeding.

\section{Discussion}

To the best of our knowledge, this is the first report regarding the use of Minirin Melt $\AA$ for the treatment of central diabetes insipidus in very young infants.
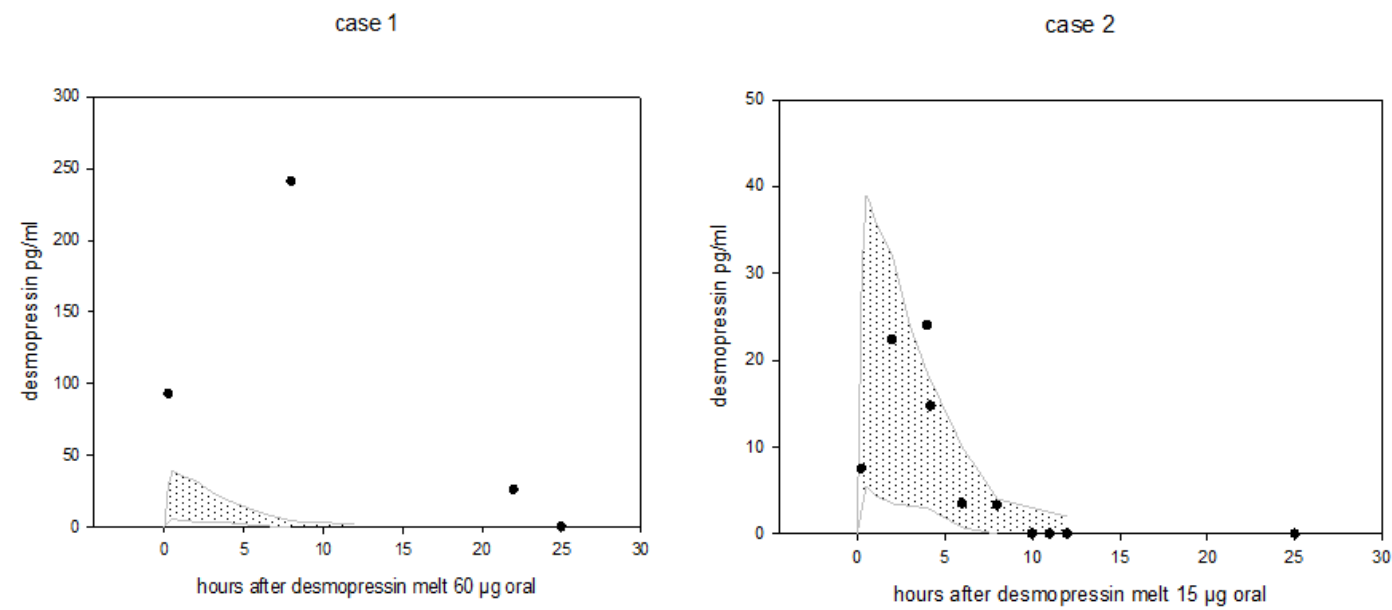

Figure 1. Pharmacokinetic Data of Desmopressin Melt Oral in Case 1 With $60 \mu$ g and Case 2 With $15 \mu$ g Plotted Against the Calculated Reference in Children With Enuresis Nocturia (6 to 12 years) at a Dose of $240 \mu \mathrm{g}$ 
A starting dose of $15 \mu \mathrm{g}$ appeared safe at this young age and a maintenance dose of 30 to $60 \mu \mathrm{g}$ daily was effective in the first two years of life. No local or systemic side effects were observed. Treatment of infants with CDI is challenging for several reasons. Infants consume almost all their calories in liquid form. To eliminate a large amount of dietary water, urine osmolality is typically low (100-150 mOsm/L) and urine output is high (5 $\mathrm{ml} / \mathrm{Kg} / \mathrm{h}$ ). Because of this obligatory high urine output, infants are prone to water intoxication if fixed antidiuresis is achieved by desmopressin treatment. Additionally, the exact volume of urine output is difficult to assess in young children, and infants are unable to both access fluids and articulate thirst to care providers. Moreover, many of these infants present with severe congenital or acquired neurological problems, leading to an impairment of their thirst mechanism. Two apparently contradictory treatment options have been proposed for young infants and very small children. Either low solute diet combined with thiazide diuretics or the use of vasopressin analogue, desmopressin (5). Low renal solute load formula reduces obligatory urinary water losses and the use of thiazide diuretics results in sodium loss, subsequent decrease of circulating volume and increase in vasoactive hormones, leading to maximal proximal sodium and water reabsorption. Many of these children would reach a steady state at higher plasma osmolality. This approach is only acceptable if hypernatremia is mild and does not coincide with neurological symptoms or failure to thrive.

Desmopressin was introduced for the treatment of CDI first in 1972 (6) and has been commercially available for many years as an intranasal solution (1972), an injectable solution for intravenous, subcutaneous or intramuscular use (1981), a nasal spray (1986) and oral solid tablet (1987) (7). Given high and fixed dosage of nasal spray, intranasal solution is more appropriate to treat CDI in young children. A major disadvantage of intranasal solution is that the absorption is highly variable and often a dilution has to be administered, making weekly preparations necessary (8). Large individual variations in absorption of dDAVP following oral administration have also been observed in children with CDI, and swallowing whole tablets remains difficult for infants and young children. Therefore, subcutaneous dDAVP acetate (4 microgram/ $\mathrm{mL}$ ) is often used in infants because of the possibility of precise administration and consistent dosing, but longterm use of subcutaneous injections is inconvenient (9). Due to variable absorption with the above mentioned formulations, it has been difficult to avoid potentially life threatening episodes of hyponatremia and fast changes of sodium levels, especially in infants. Hyponatremia results from water retention caused by drugs potent antidiuretic effect and simultaneous intake of fluids. Infants and young children are vulnerable for water intoxication and cerebral oedema, because their brain has a limited capacity to adapt with hyponatremia. This leads to a higher morbidity and mortality due to symptomatic hypona- tremia in young children compared to adults (10). We decided to treat our two young infants with sublingual desmopressin (Minirin Melt@) because of its ease of administration (the tablet was cut by a sterile stitch cutter and placed sublingually using small tweezers) and our previous experience with high day-to-day variability of the nasal solution in infants. Desmopressin oral lyophilisate (Minirin Melt $₫$ ) has been available since 2005. Oral lyophilisate formulation is well tolerated, with the same type of adverse events as reported for desmopressin tablets. Pharmacological studies demonstrated that sublingual route results in a more stable absorption compared to intranasal or oral route. Additionally, oral lyophilisate has a higher bioavailability compared to tablet, allowing lower dosing (11). In the absence of pharmacokinetic data of oral lyophilisate in neonates, size-dependent dosing schemes are absent. We observed that $60 \mu \mathrm{g}$ melt in the first case resulted in a pharmacokinetic profile that largely exceeds $(4 \mathrm{x})$ the reference frame from Osterberg of $240 \mu \mathrm{g}$ for children (6-12 years) with nocturnal enuresis. Besides, this dose covered more than 24 hours in our neonatal patient. This is against the indication of nocturnal enuresis, in which only a 12-hour coverage is required. Therefore, we started with a small dose of $15 \mu \mathrm{g}$ desmopressin in the second case, which resulted in a PK profile compatible to the reference frame. These results suggest that bioavailability characteristics are largely maintained. Despite the fact that we had to cut the tablet, we found no effect on its bioavailability (12). Minirin Melt $®$ was administered to both infants with careful monitoring of the balance between liquid intake and output. Both infants were managed with one to two daily dosing on long-term. In both infants, no episodes of hyponatremia or severe hypernatremia were reported during their first two years of life and they gained weight properly. In our experience of two cases, sublingual administration of desmopressin lyophilisate together with close followup of serum sodium and parent education about the risk of excessive fluid intake was successful and more convenient than intranasal dDAVP therapy. We recommend a starting dose of $15 \mu \mathrm{g}$ in neonates and young infants and an upward titration depending on the effect. However, more studies are required to confirm the possible use of Minirin Melt $®$ as the first line therapy in young small infants.

\section{Author's Contributions}

All authors contributed to the preparation and drafting of the manuscript.

\section{Financial Disclosure}

Raes Ann and Cools Martine reported receiving grants for speaking from Ferring. Van de Walle Johan reported receiving research grants from Ferring. The other authors had no financial interests related to the materials in the manuscript. 


\section{References}

1. Majzoub JA, Srivatsa A. Diabetes insipidus: clinical and basic aspects. Pediatr Endocrinol Rev. 2006;4 Suppl 1:60-5.

2. Baruteau J, Cartault A, Chanot A, Sevely A, Casper C. Neonatal Escherichia coli meningitis can be complicated by central permanent diabetes insipidus. J Pediatr Endocrinol Metab. 2009;22(3):213.

3. Mohn A, Schoof E, Fahlbusch R, Wenzel D, Dorr HG. The endocrine spectrum of arachnoid cysts in childhood. Pediatr Neurosurg. 1999;31(6):316-21.

4. Maghnie M, Cosi G, Genovese E, Manca-Bitti ML, Cohen A, Zecca S, et al. Central diabetes insipidus in children and young adults. $N$ Engl J Med. 2000;343(14):998-1007.

5. Rivkees SA, Dunbar N, Wilson TA. The management of central diabetes insipidus in infancy: desmopressin, low renal solute load formula, thiazide diuretics. J Pediatr Endocrinol Metab. 2007;20(4):459-69.

6. Andersson KE, Arner B. Effects of DDAVP, a synthetic analogue of vasopressin, in patients with cranial diabetes insipidus. Acta Med Scand.1972;192(1-2):21-7.
7. Vande Walle J, Stockner M, Raes A, Norgaard JP. Desmopressin 30 years in clinical use: a safety review. CurrDrug Saf. 2007;2(3):232-8.

8. Fjellestad-Paulsen A, Paulsen O, d'Agay-Abensour L, Lundin S, Czernichow P. Central diabetes insipidus: oral treatment with dDAVP. Regul Pept. 1993;45(1-2):303-7.

9. Blanco EJ, Lane AH, Aijaz N, Blumberg D, Wilson TA. Use of subcutaneous DDAVP in infants with central diabetes insipidus. $J$ Pediatr Endocrinol Metab. 2006;19(7):919-25.

10. Thumfart J, Roehr CC, Kapelari K, Querfeld U, Eggert P, Muller D. Desmopressin associated symptomatic hyponatremic hypervolemia in children. Are there predictive factors? J Urol. 2005;174(1):294-8.

11. Steiner IM, Kaehler ST, Sauermann R, Rinosl H, Muller M, Joukhadar C. Plasma pharmacokinetics of desmopressin following sublingual administration: an exploratory dose-escalation study in healthy male volunteers. Int J Clin Pharmacol Ther. 2006;44(4):1729.

12. Vande Walle JG, Bogaert GA, Mattsson S, Schurmans T, Hoebeke P, Deboe $V$, et al. A new fast-melting oral formulation of desmopressin: a pharmacodynamic study in children with primary nocturnal enuresis. BJU Int. 2006;97(3):603-9. 